Editorial:

\title{
Technology enhanced learning, research impact, and open scholarship
}

Thomas Cochrane

Auckland University of Technology

Petrea Redmond

University of Southern Queensland

\author{
Linda Corrin \\ University of Melbourne
}

\begin{abstract}
This guest editorial explores the potential impact of alternative metrics and social research networks to enhance technology enhanced learning (TEL) research and practice, particularly in the realm of open scholarship. Conventional measures of research impact are based upon a publications' impact factor. Article or author-level metrics can provide a better view of the contribution to the field of research of a specific research project. However, metrics are based upon citation counts can take years to accumulate. This can result in a significant lag between the original research, communicating research outcomes to the wider research community, and research impact. TEL researchers are in a unique position to spearhead the growing movement towards open social scholarship and alternative metrics. Alternative metrics provide a powerful addition to conventional measures of research impact, facilitating a dynamic conversation around TEL research. Social media conversations provide the foundation for a collaborative open scholarship of technology enhanced learning as both an enhancement and alternative to conventional modes of research impact measurement. This guest editorial outlines key tools currently used to measure impact. We argue that a culture of open scholarship enhanced via social media and measured via alternative metrics creates an effective feedback loop between research and practice.
\end{abstract}

\section{Introduction}

Citation counts and the impact factor of the journals in which they have published are conventional methods for measuring a researcher's academic credibility. However, citation counts in subscription-based journals can take years to accumulate into a significant article or author-level impact factor. A focus upon achieving high impact factor ratings and high citation counts rather than practical impact on society beyond the academic niche has led to criticism of these measures of journal rank as a self-perpetuating game (Brembs, Button, \& Munafò, 2013; Cogent OA, 2015; Ferrara \& Romero, 2013; The PLoS Medicine Editors, 2006).

The open access publication movement has shown that citation counts are significantly increased when articles are published in open access journals, implying that the reach of research published in such journals is wider than the relatively small communities of academics with subscription-based access to closed publications (Gargouri et al., 2010). AJET is well placed in this regard, as one of the top-ranked open access journals in the domain of technology enhanced learning (TEL). However, citation count statistics are only one measure of the impact of TEL research. Social scholarship has been growing alongside the open access movement, yet there is still limited awareness within the TEL research community of alternative metrics. Alternative metrics attempt to measure research impact through mentions in dynamic social media conversations linked to the research artefacts, providing a powerful addition to citation-based measures of research impact. Alternative metrics badges are now integrated into many research repository and journal metrics. In this guest editorial we attempt to demystify research impact factors by outlining some of the conventional impact metrics, and to explore how alternative metrics and social media can both enhance these impact factors and provide evidence of wider impact. In doing so, we (and the AJET editorial team) are not promoting or endorsing specific tools or approaches, rather we are profiling a landscape of options to raise TEL researchers' awareness of ways to measure and/or promote the impact of their work. This information is also relevant for other academic researchers. 


\section{Demystifying alternative metrics and open scholarship}

There are four basic measures of research impact in the digital age (Priem, Taraborelli, Goth, \& Neylon, 2010): usage (via download statistics), peer-review (acceptance and publication in scholarly publications), citations (the number of times an article is cited in other published articles), and alternative metrics (capturing social media mentions and conversations around scholarly publications). In the following sections we explore the relationship between these measures of research impact and demystify the various key terms.

\section{Research metrics and impact factor}

Conventional measures (metrics) for the impact of research journals and books are based upon the average citation statistics accrued over time, rather than upon individual journal articles and book chapters. Articlelevel metrics provide a way of analysing an author's impact rather than the publication in general. Hirsch (2005) proposed the index of scientific research impact or h-index as an author-level metric to measure relative research quality between scholars in the same contexts. The h-index provides a more granular approach than total citation or publication counts to reflect the number of an author's publications and the number of citations per publication, that is, an h-index of 11 means that you have 11 papers with at least 11 citations. Thus, the h-index attempts to provide a better measure of research quality than merely a count of either publication numbers or citation counts alone. Criticisms of the h-index include the impact of selfcitations (Ferrara \& Romero, 2013) and its limited added value to the accuracy of impact factors. However, the h-index has found acceptance among the main research databases as one measure of author-level research impact. TEL research publications are generally categorised under social sciences/educational technology. The largest academic research bibliography and citation databases in these fields are Google Scholar, Web of Science, and Scopus. These research databases provide interactive h-index graphs and other research metric analytics for author outputs found within their respective databases. While there is overlap of content between the three databases, a comprehensive view of research impact requires a search of all three. Google Scholar is open access, whereas Scopus and Web of Science are subscription-based services. All three provide free access to author analytics, but with different results.

The Web of Science (http://wokinfo.com/) database, was formally the ISI Web of Knowledge, and is owned by Thomson-Reuters. It is one of the largest databases of research publications and citation indexes, covering over 18,000 high impact journals, over 180,000 conference proceedings, and over 80,000 books from around the world (Web of Science, 2018). Web of Science article and author-level analytics include citation metrics, author collaborative network, and citing articles network.

The Scopus (https://www.scopus.com/) academic database indexes peer-reviewed publications from nearly 23,700 titles from over 5,000 publishers, including journals, books and conference proceedings. It is now owned by the Elsevier publishing company yet claims to be unbiased in its coverage due to implementing an independent content selection and advisory board (Scopus, 2018).

Google Scholar (https://scholar.google.com.au/) aims to index all academic knowledge. In 2014, it was estimated to include around 160-165 million documents, however the exact size of its database is uncertain (Orduna-Malea, Ayllón, Martín-Martín, \& Delgado López-Cózar, 2015). Established in 2004, Google Scholar provides an overview of an author's citation count, h-index, and their own i10-index, and has grown to be the most exhaustive database of academic publications and research metrics (Martin-Martin, OrdunaMalea, Harzing, \& Delgado López-Cózar, 2017; Orduna-Malea, Martín-Martín, \& Delgado López-Cózar, 2017). In 2011, Google introduced the i10-index to indicate the number of publications an author has published that have received 10 or more citations. Google Scholar metrics also provides metrics ranking of the impact of academic journals. The main criticism of Google Scholar has been the credibility of the journals indexed in the database, which includes both recognised academic journals and also journals that have been identified as predatory. Predatory journals typically solicit publication of research articles from authors, promising quick review and publication times, while evidencing little or no academic integrity or quality and sometimes requiring payment (Weller, 2014). 


\section{Researcher IDs}

Researcher IDs enable a researcher to collate a portfolio of research publications across the wide diversity of journals, book chapters, and conference proceedings that they have published in. Researcher IDs also enable a researcher to lay claim to their research published under different varieties of their name and across the various institutions at which they have worked. There are four main researcher IDs: Scopus author ID, researcherID, ORCID, and Google Scholar ID. Both Scopus and Web of Science provide authors with the option of creating a researcher ID based upon the publications recorded within their respective databases. Individual articles are indexed within these publication databases by their unique digital object identifier (DOI), which is a fixed document identifier, unlike a URL that may change.

The Open Researcher and Contributor ID (ORCID) is a recent attempt at enabling an independent open researcher ID that integrates directly with both Scopus ID and ResearcherID databases. ORCID was launched in 2012 and has received rapid uptake, reaching over 4.8 million registered users (ORCID, 2018). ORCID (https://orcid.org/) facilitates import and export of research data and researcher profiles between an ORCID profile, Scopus author ID, ResearcherID, and Publons (https://publons.com/home/) (a repository of peer review records), creating an independent consolidated research profile. ORCID also provides a facility for generating a QR code to enable quick sharing of research profiles via mobile devices. Most institutional research databases now contain a field for a researcher to add their ORCID to their institutional research profile.

The Scopus author ID utilises the Scopus database to curate a researcher ID, providing a freely accessible researcher and publication search tool (http://www.scopus.com/search/form/authorFreeLookup.uri?null). A Scopus author ID provides a summary of research analytics for an individual researcher based upon the Scopus database, including: h-index, total citations, and an interactive citation graph over time.

ResearcherID utilises the Web of Science database (www.researcherid.com/) and provides researcher publication search and analytics similar to Scopus (www.researcherid.com/ViewProfileSearch.action). Web of Science provides additional researcher tools such as ResearcherID badges and citation maps.

In 2012, Google added scholar citation profiles (https://scholar.google.com/citations), enabling individual researchers to curate a citation profile based upon Google's extensive database of publications. Google Scholar provides authors with a range of research metrics but is still not as highly regarded as ResearcherID or Scopus author ID profiles within academia, because it includes non-scholarly works and double counting (Harzing \& van der Wal, 2009), and does not provide self-citation filtering.

\section{Social research networks}

Social research networks enable researchers to become part of a global research community to share, discuss and disseminate their research, and provide another measure of research impact. This is an underresearched area of open scholarship (Manca, 2018). There are four leading social research networks: ResearchGate, Academia.edu, LinkedIn, and Mendeley. These research networks are all accessible via mobile devices through either a mobile app or a mobile web interface, and they integrate with mobile social media networks (Twitter, Google+, Facebook) for sharing research updates. Having a profile presence on each of these social research networks enables a researcher to reach a variety of audiences, potentially creating a wider impact for their research publications. Closely related to these social research networks is Publons, that is a social network for curating review and editorial activity. This list of networks and repositories is indicative rather than exhaustive, with others emerging, and some more popular in different countries than others.

ResearchGate (http://researchgate.net/) is essentially a social network for academic researchers. ResearchGate provides a mix of impact factor analytics, including a cumulative publication level impact factor, article-level statistics (downloads and reads), author-level statistics via a ResearchGate score, and promotes discussion around research via a network of followers of other researchers. ResearchGate encourages users to upload and share their research articles as long as the publisher copyright allows this, therefore ResearchGate works particularly well with open access publications. It also enables the private sharing of publications. Additionally, ResearchGate provides the option of creating a DOI for an uploaded research article if it does not already have one - effectively providing researchers with a self-publishing option. 
Academia.edu (https://www.academia.edu/) provides a researcher with an alternative institutional research profile, research dissemination channel, and participation within a social network of similar researchers. Academia.edu metrics include citations plus reader and article download statistics. In 2013, Elsevier issued take down notices to Academia.edu referring to Elsevier copyrighted research articles uploaded and shared on the Academia.edu site. This hardline approach resulted in some academics boycotting Elsevier publications and others boycotting Academia.edu (Solon, 2013).

LinkedIn (https://au.linkedin.com/) was initially created as a professional online portfolio and employment network. However, LinkedIn has expanded to include research profiles by allowing users to add publications and projects to a LinkedIn profile. LinkedIn was purchased by Microsoft in 2016 (Microsoft News Center, 2016).

Mendeley (https://www.mendeley.com) is a cross between an online reference library manager and a research sharing community. Mendeley provides synchronisation between a desktop client application, an iOS/Android mobile app, and an online account. Mendeley provides research metrics based around article reads and is introducing Scopus publications and statistics. In 2013, Mendeley was purchased by the large publishing house, Elsevier (Shaw, 2013).

Publons (https://publons.com/) provides an opportunity for academics to create and share a record of their reviewing and editorial activity that are professional activities in which many academics engage, yet it is often an invisible aspect of academic workload. A Publons profile includes academic review activity, editorial board memberships and editorial activity, with comparative statistical analysis to other Publons users. When journals subscribe to Publons reviewing and editorial activities related to the journal is automatically updated. It also allows individual users to create accounts for free and to register their activity with non-subscribing journals manually. Publons integrates with ORCID and ResearcherID to create a consolidated research activity profile.

\section{Open access publications}

The growth of open access journals is transferring power long held by a select number of large publishing companies over the access and distribution of scholarship. Increasingly government funding agencies and institutions are expecting publicly funded research to be publicly available via open access. The impact of this trend is demonstrated by the inclusion of several full open access peer reviewed journals within the top 10 educational technology journals by Google Scholar (2018). At the time of writing these journals include: Journal of Educational Technology \& Society, International Review of Research in Open and Distributed Learning, and the Australasian Journal of Educational Technology. An indication of the growth of open access publication models (Olijhoek, 2015) is the development of open access directories such as the Directory of Open Access Journals (https://doaj.org/), and user-generated directories such as an open database of open access educational technology journals curated by Veletsianos (2009). Weller (2014) argues that the battle for open scholarship has already been won but warns that it may not look like the type of openness that is characterised by the eight principles that he promotes: (1) freedom to reuse, (2) open access, (3) free cost, (4) easy use, (5) digital networked content, (6) social community-based approaches, (7) an ethical approach, (8) and an efficient model.

\section{Alternative metrics}

One of the changes in the current research environment is the ability to track and demonstrate the reach and influence of research. This reach and influence also includes that of social media. Alternative metrics are non-conventional metrics based on the Social Web for analysing and displaying scholarship about research. A current tool which measures social media impact is Altmetrics (https://www.altmetric.com/). In 2010, the Altmetrics tool was introduced by Priem et al. (2010) in a founding manifesto and defined as "the study and use of scholarly impact measures based on activity in online tools and environments." (Priem, Groth \& Taraborelli, 2012, p. 2). Altmetrics aims to integrate the use of social media tools into the practice of professional communities to "inform new, broader, faster measures of impact, complementing traditional citation metrics” (Priem, Piwowar \& Hemminger, 2012). Altmetrics utilise a range of social media networks to measure the conversation generated and impact of research beyond the limitations of conventional academic publishing (Williams \& Padula, 2015). It does this by measuring the impact across social media networks, including: Twitter, Facebook, Google+, Pinterest, Blogs, Mendeley, CiteULike, 
Reddit, Wikipedia, Reviews on F1000, YouTube, PubPeer, and Publons. A numerical score is attached to a research article based upon the combined mentions of the article DOI across the various social media channels curated by Altmetrics. Altmetrics provides comparative statistics for research impact for similar aged articles within a particular publication, a map of the social media conversation around an article, and links to view the social media citations in context. Altmetrics is not targeted at replacing research metrics such as the h-index, but in augmenting conventional metrics it provides a wider view of the impact of an author's research at the article level.

To use the service, publications must subscribe to Altmetrics, and a growing number of both subscriptionbased journals and open access journals now subscribe to Altmetrics providing added value analytics to the researchers publishing in these journals. Many institutional research database systems now include Altmetrics figures alongside the publication impact factor, as well as the article Scopus and Web of Science scores. Altmetrics are generated much more dynamically and quicker than conventional academic published citation metrics. In our option there is a direct relationship between Altmetrics and TEL research, as the social media networks analysed have become drivers of conversation around research publications on these networks. Social media can be used to raise awareness of academic research articles, provide links between related research, and increase citations (Terras, 2012). However, social media networks can also be misused to drive up counts, may be impacted by the commercial providers of the social media services, and are heterogeneous and constantly changing which makes generalisation of impact difficult (Bornmann, 2014; Haustein, 2016; Sugimoto, Work, Larivière, \& Haustein, 2017). Issues like these have caused concerns about the ability of Altmetrics to measure more than just the popularity of publication, instead to reflect the quality of the research contained in the publication.

An example of how Altmetrics can enhance research metrics by providing a fuller view of an article's impact is the comparison between the conventional citation counts and Altmetrics rank for an article by Brembs et al., (2013) that criticises journal rank. The article has accumulated 89 citations reported via Scopus (http://bit.ly/1QH7EeP), yet has an impressive Altmetrics score of 1125 (http://www.altmetric.com/details/1527978\#score), with 133674 reads and 9450 downloads from the open access publisher site (https://doi.org/10.3389/fnhum.2013.00291). The Altmetrics score is derived from a combination of: 17 news outlets, 1020 tweeters, 2 peer review sites, 48 Facebook pages, 66 Google+ users, 641 Mendeley readers, 6 CiteULike mentions, 38 blog posts, 7 Redditors, 1 research highlight platform, and 2 Q\&A threads. The article therefore demonstrates a significant impact on social media.

Despite the disadvantages indicated previously, as alternative metrics mature we argue for the integration of social media and to empower open scholarship to increase scholarly conversations and enhance conventional measures of research impact and reflective practice. Alternative and conventional metrics can work hand in hand to generate a broader audience of readers and higher overall citation rates, increasing the possible impact of the research and they make researchers work more visible.

In summary there are multiple elements of impact factor that make up an academic researcher's overall impact profile. The impact of academic research can be defined by a number of different elements:

1. Journal elements: including relative rankings, for example Scopus, Web of Science, SJR;

2. Article elements: including citations, reads, downloads, and alternative metrics;

3. Author ID elements: including Scopus author ID, ResearcherID, ORCID, and Google Scholar ID; and

4. Social Research Networks elements: such as ResearchGate, Academia.edu, LinkedIn, Mendeley, and Publons.

\section{Conclusion and recommendations}

The AJET editorial team have begun exploring how AJET can critically engage with social media research networks and alternative metrics. We have created an AJET Twitter account (@AJET_eds) and an associated hashtag, and encourage AJET authors and reviewers to add ORCID and social research network profiles to their AJET profiles. We recommend TEL researchers critically engage with the variety of emerging social media research networks and create consolidated research profiles via ORCID. These simple activities will influence conventional and emerging research impact factors to increase the impact of innovation in teaching and learning. 
In this guest editorial we have attempted to demystify research impact factors for researchers. The growth of open access research publications, research social networks, and alternative metrics presents a unique opportunity for TEL researchers to leverage these networks to provide enhanced evidence of the impact of their research.

\section{In this issue}

This issue includes papers that contribute practically and theoretically to discussions about TEL in higher education. The 10 papers are produced by 42 scholars from 10 different countries. The first paper in this issue, "Social presence and motivation in online environments: Second life versus the enocta learning management system/adobe connect” by Ozonu, Yanpar-Yelken, and Sancar-Tokmak, compares the social presence of students in an LMS and Second Life. Using a sociability scale and motivation questionnaire they found there were significant differences in the students' social presence and motivation for study. Next Teräs and Kartoğlu focus on authentic learning within online professional development in "Authentic learning with technology for professional development in vaccine management”. Their paper describes a pilot study exploring how authentic online learning could support the development of transferable professional skills.

In the only single authored paper of this issue, "The influence of structured guidance on pre-service teachers' blog writing”, Lee reports on a structured blogging project with pre-service teachers. The article notes that the pre-service teachers were more likely to provide feedback to their peers that was social, affirming and reflective. In "Bot-teachers in hybrid massive open online courses (MOOCs): A posthumanist experience” the authors, Bozkurt, Kilgore, and Crosslin examine third generation hybrid MOOCs and the use of bot-teachers. Using the lenses of the community of inquiry (CoI) and actor-network theory (ANT) they found that bot-teachers can effectively facilitate and increase online discussion.

The paper, “A clinical educator's experience using a virtual patient to teach communication and interpersonal skills” by Bánszki, Beilby, Quail, Allen, Brundage, and Spitalnick, investigates a course examiner's experience when their students interact with a virtual patient in a virtual learning environment. The results indicate that working in the virtual learning environment required the course examiner to modify their pedagogical role. "Comparing active learning techniques: the effect of clickers and discussion groups on student perceptions and performance” the American authors Walker, Spangler, Lloyd, Walker, Wessels, and Summerville compared student perspectives on the use of clickers with student discussion groups. Their findings revealed that students preferred the clicker approach rather than structured, student-led discussion groups.

The next article, "Physiotherapy student perspectives on synchronous dual-campus learning and teaching" explores student perceptions of synchronous dual-campus delivery of a physiotherapy programme. Divanoglou, Chance-Larsen, Fleming and Wolfe identified potential benefits for students by accessing expertise from both campuses along with difficulties related to cross-campus communication, logistics, and opportunities for interaction and engagement. Following this, in "Examining the relationship between English language learners' online self-regulation and their self-efficacy” Su, Zheng, Liang, and Tsai explore EFL learners' online self-regulation using online self-regulation and English language self-efficacy questionnaires. The articles notes there are reciprocal relationships between EFL students' online selfregulation and their English self-efficacy, and future research will investigate the possible bi-directional nature of the relationships between self-regulation and self-efficacy.

"Learning analytics experience among academics in Australia and Malaysia: a comparison” by West, Tasir, Si Na, Toohey, Abdullah, Searle, Jumaat, and Price, makes a comparison of the experiences of academics in the use of learning analytics across the two countries. They found that academics in both countries have an interest in learning analytics, however it is to improve teaching rather than the institutional concern of retention. To end this issue, an article by Costa, Murphy, Pereira, and Taylor, "Higher education students' experiences of digital learning and (dis)empowerment” explores higher education students' sense of agency when participating online. On one hand the students felt they were able to more freely develop a voice online when compared to face-to-face, yet the visibility of their voice online led them to feel a sense of vulnerability. 


\section{Acknowledgements}

The guest authors of this editorial would like to thank the lead editors for the opportunity to raise awareness of the important role social media research networks can play in promoting and enhancing research impact. As lead editors, Associate Professor Eva Heinrich, Associate Professor Michael Henderson, and Associate Professor Petrea Redmond, work with a committed team of associate editors who facilitate the reviews and author revisions of papers: Associate Professor Shirley Agostinho; Dr Thomas Donald Cochrane; Dr Linda Corrin; Dr Helen Farley; Associate Professor Paul Gruba; Professor Judi Harris; Dr Jason M Lodge; Associate Professor Lina Markauskaite; Dr Stephen Marshall; and Dr Michael Phillips. Backing up the editorial team we have two dedicated copyeditors, Kayleen Wood and Antonina Petrolito who work closely with authors to enhance the quality of the articles by ensuring the text is concise, consistent, and accurate. We also need to thank our large number of expert reviewers who ensure our articles are of high standard. And finally, thank you to the authors, who offer valuable new understandings in the field of educational technology; and the readers, without you there would be no AJET.

\section{References}

Bornmann, L. (2014). Do altmetrics point to the broader impact of research? An overview of benefits and disadvantages of altmetrics. Journal of Informetrics, 8(4), 895-903. https://doi.org//10.1016/j.joi.2014.09.005

Brembs, B., Button, K., \& Munafò, M. (2013). Deep Impact: Unintended consequences of journal rank. Frontiers in Human Neuroscience, 7(Article 291), 1-12. https://doi.org//10.3389/fnhum.2013.00291

Cogent OA (2015). Why is impact factor so important? Knowledge Base. Retrieved from http://explore.cogentoa.com/knowledge-base/why-is-impact-factor-so-important

Ferrara, E., \& Romero, A. (2013). Scientific impact evaluation and the effect of self-citations: Mitigating the bias by discounting the h-index. Journal of the American Society for Information Science and Technology, 64(11), 2332-2339. https://dx.doi.org/10.1002\%2Fasi.22976

Google Scholar (2018). Top publications - Educational Technology. Retrieved from https://scholar.google.co.nz/citations?view_op=top_venues\&hl=en\&vq=soc_educationaltechnology

Gargouri, Y., Hajjem, C., Larivière, V., Gingras, Y., Carr, L., Brody, T., \& Harnad, S. (2010). Selfselected or mandated, open access increases citation impact for higher quality research. PloS one, 5(10), e13636.

Harzing, A.W. \& van der Wal, R. (2009). A Google Scholar h-index for journals: An alternative metric to measure journal impact in Economics \& Business? Journal of the American Society for Information Science and Technology, 60(1), 41-46.

Haustein, S. (2016). Grand challenges in altmetrics: Heterogeneity, data quality and dependencies. Scientometrics, 108(1), 413-423.

Hirsch, J. E. (2005). An index to quantify an individual's scientific research output. Proceedings of the National Academy of Sciences, 102(46), 16569-16572.

https://dx.doi.org/10.1073\%2Fpnas.0507655102

Manca, S. (2018). ResearchGate and Academia.edu as networked socio-technical systems for scholarly communication: A literature review. Research in Learning Technology, 26, 1-16. https://doi.org//10.25304/rlt.v26.2008

Martin-Martin, A., Orduna-Malea, E., Harzing, A.-W., \& Delgado López-Cózar, E. (2017). Can we use Google Scholar to identify highly-cited documents? Journal of Informetrics, 11(1), 152-163. https://doi.org/10.1016/j.joi.2016.11.008

Microsoft News Center (2016). Microsoft to acquire LinkedIn. Retrieved from https://news.microsoft.com/2016/06/13/microsoft-to-acquire-linkedin/

Olijhoek, T. (2015). Directory of Open Access Journals. Retrieved from https://doaj.org

ORCID (2018). ORCID Statistics. Retrieved from https://orcid.org/statistics

Orduna-Malea, E., Ayllón, J., Martín-Martín, A., \& Delgado López-Cózar, E. (2015). Methods for estimating the size of Google Scholar. Scientometrics, 104(3), 931-949.

https://doi.org//10.1007/s11192-015-1614-6

Orduna-Malea, E., Martín-Martín, A., \& Delgado López-Cózar, E. (2017). Google Scholar as a source for scholarly evaluation: A bibliographic review of database errors, Revista Española de Documentación Científica, 40(4). https://doi.org//10.3989/redc.2017.4.1500

Priem, J., Groth, P., \& Taraborelli, D. (2012). The Altmetrics Collection. PLOS One, 7(11), e48753. https://doi.org//10.1371/journal.pone.0048753 
Priem, J., Piwowar, H., \& Hemminger, B. (2012, March). Altmetrics in the wild: Using social media to explore scholarly impact. Paper presented at the Altmetrics12: ACM Web Science Conference 2012 Workshop, Evanston, IL. http://altmetrics.org/altmetrics12/priem/

Priem, J., Taraborelli, D., Goth, P., \& Neylon, C. (2010). Altmetrics: A manifesto. Retrieved from http://altmetrics.org/manifesto/

Scopus. (2018). Content. Retrieved from https://www.elsevier.com/solutions/scopus/content

Shaw, C. (2013, April 10). Elsevier buys Mendeley: Your reaction [Blog post]. Retrieved from https://www.theguardian.com/higher-education-network/blog/2013/apr/10/elsevier-buys-mendeleyacademic-reaction

Solon, O. (2013). Elsevier clamps down on academics posting their own papers online. Retrieved from http://www.wired.co.uk/article/elsevier-versus-open-access

Sugimoto, C. R., Work, S., Larivière, V., \& Haustein, S. (2017). Scholarly use of social media and altmetrics: A review of the literature. Journal of the Association for Information Science and Technology, 68(9), 2037-2062.

Terras, M. (2012). The impact of social media on the dissemination of research: Results of an experiment. Journal of Digital Humanities, 1(3). Retrieved from http://journalofdigitalhumanities.org/1-3/theimpact-of-social-media-on-the-dissemination-of-research-by-melissa-terras/

The PLoS Medicine Editors (2006). The impact factor game. PLoS Med, 3(6), e291. https://doi.org//10.1371/journal.pmed.0030291

Veletsianos, G. (2009). Open access educational technology journals. Retrieved from http://www.veletsianos.com/2009/08/09/open-access-educational-technology-journals/

Web of Science (2018). Web of Science: The complete citation connection. Retrieved from http://wokinfo.com/citationconnection/realfacts/ - regional

Weller, M. (2014). The battle for open: How openness won and why it doesn't feel like victory. London: Ubiquity Press.

Williams, C., \& Padula, D. (2015). The evolution of impact factors: From bibliometrics to altmetrics. Retrieved from http://docs.scholastica.s3.amazonaws.com/altmetrics/evolution-of-impactindicators.pdf

Corresponding author: Thomas Cochrane, thomas.cochrane@aut.ac.nz

Australasian Journal of Educational Technology (C) 2018.

Please cite as: Cochrane, T., Redmond, P., \& Corrin, L. (2018). Editorial: Technology enhanced learning, research impact, and open scholarship. Australasian Journal of Educational Technology, 34(3), i-viii. https://doi.org/10.14742/ajet.4640 